\title{
42. A Novel Point Mutation of the RET Proto-oncogene in Small Cell Lung Carcinoma Cell Lines
}

\author{
By Hitoyasu Futami, Shin-ichi Egawa, and Ken Yamaguchi \\ Growth Factor Division, National Cancer Center Research \\ Institute, 5-1-1 Tsukiji, Chuo-ku, Tokyo 104 \\ (Communicated by Takashi Sugimura, M. J. A., Dec. 12, 1994)
}

\begin{abstract}
Point mutations of the RET proto-oncogene are associated with the development of inherited diseases including multiple endocrine neoplasia (MEN) type 2, familial medullary thyroid carcinoma (MTC), and Hirschsprung's disease as well as a part of sporadic MTCs and pheochromocytomas. In the present study, we examined point mutations of the RET proto-oncogene in exons 10,11 and 16 in small cell lung carcinoma (SCLC) cell lines. A novel point mutation from GCC to GAC at codon 664 in exon 11 was identified in 2 out of 6 SCLC cell lines; this alteration results in an amino acid substitution of aspartic acid for alanine. This point mutation was not detected in other types of cancer cell lines so far examined. Point mutations of the RET proto-oncogene reported previously in exons 10, 11 and 16 in above-mentioned diseases were not detected in the SCLC cell lines. These results suggest that this point mutation of the RET proto-oncogene is closely associated with a part of SCLC.
\end{abstract}

Key words: Small cell lung carcinoma; RET; point mutation; multiple endocrine neoplasia; medullary thyroid carcinoma.

Introduction. The RET proto-oncogene is thought to encode a receptor tyrosine kinase for an unidentified ligand. ${ }^{1,2)}$ Rearrangements of the $R E T$ proto-oncogene were reported in human papillary thyroid carcinoma. ${ }^{3), 4)}$ More recently, it was reported that a new rearrangement occurred at the same position by the fusion of the $R E T$ tyrosine kinase domain with part of the regulatory subunit $\mathrm{I} \alpha$ of protein kinase $\mathrm{A}$ in papillary thyroid carcinoma. $^{5)}$ In addition, overexpression of this gene was observed in human neuroblastomas. ${ }^{6), 7)}$ Very recently, germ-line point mutations of the $R E T$ proto-oncogene have been reported to be closely associated with the development of several inherited diseases including multiple endocrine neoplasia type 2A (MEN2A) ${ }^{8), 9)}$ and $2 \mathrm{~B}(\mathrm{MEN} 2 \mathrm{~B}),{ }^{10)}$ familial medullary thyroid carcinoma (FMTC), ${ }^{8}$ and Hirschsprung's disease. ${ }^{12), 13)}$ Furthermore, somatic mutations of the RET proto-oncogene were detected in some sporadic cases of medullary thyroid carcinoma (MTC) and pheochromocytoma. ${ }^{10), 11)}$ Thus, point mutations of the RET proto-oncogene may play an important role in the carcinogenesis of MTC and pheochromocytoma.

Small cell lung carcinoma (SCLC) is an aggressive and rapidly growing neoplasm which tends to be disseminated to systemic organs by the time of diagnosis. Although the growth rate and metastatic potential are quite different between SCLC and MTC, they share similar biological characteristics of neuroendocrine tumors. Our previous studies revealed the close relationship between these two morbidities in terms of peptide hormone production; both cancers tend to produce calcitonin and gastrin-releasing peptide, concomitantly. ${ }^{14), 15)}$ In the present study, we examined whether SCLC cell lines possessed point mutations of the RET proto-oncogene, as was the case of MTC, and found a novel point mutation in two SCLC cell lines. 
Materials and methods. Cell Lines. Four SCLC (Lu130, Lu134A, Lu135, Lu139) and 2 non-SCLC (Lu65, Lu99) cell lines were established at National Cancer Center Research Institute. Two SCLC (H69 and N231) cell lines were established at National Cancer Institute, USA. An MTC cell line (TT) was obtained from the American Type Culture Collection (Rockville, MD, USA). These cell lines were maintained as previously described. ${ }^{16)}$ Furthermore, 8 human pancreatic cancer cell lines, 1 human gastric cancer cell line, and 2 human neuroblastoma cell lines were used in this study.

Polymerase chain reaction single-strand conformation polymorphism (PCRSSCP). Genomic DNA was extracted from cells by proteinase K/RNase digestion and phenol/chloroform extraction. Mutations in genomic DNA were screened by PCR-SSCP essentially as previously described. ${ }^{17)}$ PCR primers were designed for the three exons where mutations were identified in MEN2A and MEN2B. The primer sequences were based on the published cDNA sequence (GenBank accession numbers X12949, and $\mathrm{X} 15262$ ) and exon-intron boundaries, ${ }^{18)}$ and were as follows. Exon 10: (forward) 5'-CTACGTCTGCCTCAGGGGGC-3'， (reverse) 5'-GCCACCCACTCACCCTGGAT-3'; Exon 11: (forward) 5'-ACCCCCACCCACAGATCCAC-3', (reverse) 5'-GAAGAGGAGTAGCTGACCGG-3'; Exon 16: (forward) 5'-AGAGAGTAGAGTAACTTCAATGTC-3', (reverse) 5 '-CTACATGTATAAGGGTGTTT- 3 '. The primers were labeled with $\left[\gamma_{-}{ }^{32} \mathrm{P}\right] \mathrm{PTP}$ $(>5,000 \mathrm{Ci} / \mathrm{mmol}$, Amersham, Tokyo) and T4 polynucleotide kinase (10 units, Nippon Gene Ltd., Toyama). PCR was carried out by one cycle of denaturation at $94^{\circ} \mathrm{C}$ for $3 \mathrm{~min}$, annealing at $56^{\circ} \mathrm{C}$ for exon 16 , or $68^{\circ} \mathrm{C}$ for exons 10 and 11 for 2 min, and extension at $72^{\circ} \mathrm{C}$ for $3 \mathrm{~min}$ followed by 25 cycles of denaturation for $1 \mathrm{~min}$, annealing for $1.5 \mathrm{~min}$, and extension for $1.5 \mathrm{~min}$ with an automated thermal cycler "RoboCycler Gradient" (Stratagene, La Jolla, CA, USA). Samples denatured at $95^{\circ} \mathrm{C}$ for 5 min were run in $6 \%$ non-denaturing polyacrylamide gels containing $10 \%$ glycerol at room temperature. DNA bands on the gel were visualized by autoradiography.

Sequence analysis. Nucleotide sequences of the amplified DNA fragments were analyzed by direct sequencing of the PCR products using a Sequenase PCR product sequence kit (United States Biochemical, Cleveland, OH, USA) according to the manufacturer's instructions. The sequencing primer was as follows: 5'-GCGGGCCTCCGGAAGGT-3'.

Results. The RET proto-oncogene in 6 SCLC, 2 non-SCLC, and TT cell lines were examined by PCR-SSCP analysis for point mutations which are associated with MEN2A, MEN2B, FMTC, sporadic MTC, and sporadic pheochromocytoma. Since all point mutations reported so far in these diseases were detected in exons 10, 11, and 16, PCR primers were designed to amplify these regions. The expected PCR products for exons 10 , 11 and 16 were 148, 204, and 175 base pairs in length, respectively. Single-strand conformational variants (SSCVs) were observed only in the PCR products of exon 11 of 2 SCLC cell lines, Lu130 and Lu134A (Fig. 1). The normal PCR product of exon 11 was not observed in these two cell lines, suggesting the absence of the normal allele. SSCV was also detected in TT, an MTC cell line, but its pattern was different from those of 2 SCLC cell lines. The normal band was identified in TT. SSCV was not detected in the PCR products of exons 10 and 16 in any of the cell lines. In addition, SSCV was not identified in the PCR products of exons 10, 11, and 16 in other 11 human cancer cell lines.

Direct sequencing of the PCR products showing SSCV revealed that exon 11 of the RET proto-oncogene in Lu130 and Lu134A possessed the point mutation from GCC to GAC at codon 664 (Fig. 2); codon number was based on the revised report of amino acid sequences of the RET proto-oncogene. ${ }^{19)}$ This nucleotide alteration results in the substitution of aspartic acid (Asp) for alanine (Ala). Normal sequences were not detected at codon 664 in the two cell lines, indicating the absence of the normal allele in these cells. 


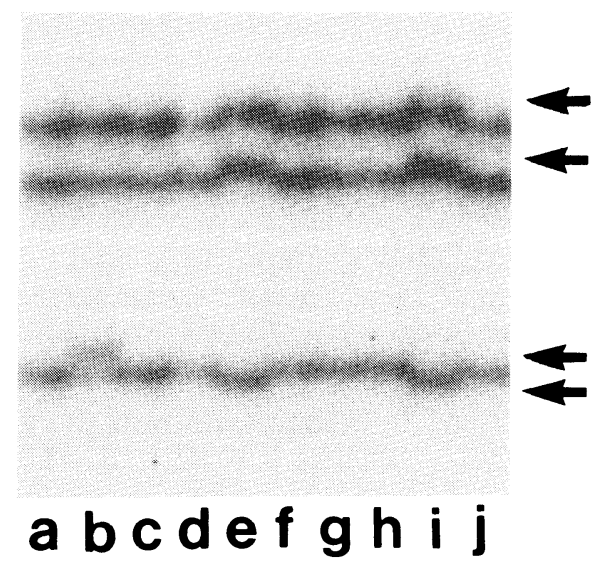

Fig. 1. SSCP analysis of the RET proto-oncogene in exon 11. The arrows indicate the abnormal bands on the lanes of $b$, e, and i. Lanes indicate samples prepared from the following cells: a, peripheral blood mononuclear cells (PBMC) from a normal volunteer; b, TT; c, H69; d, N231; e, Lu134A; f, Lu135; g, Lu99; h, Lu139; i, Lu130; j, Lu65.

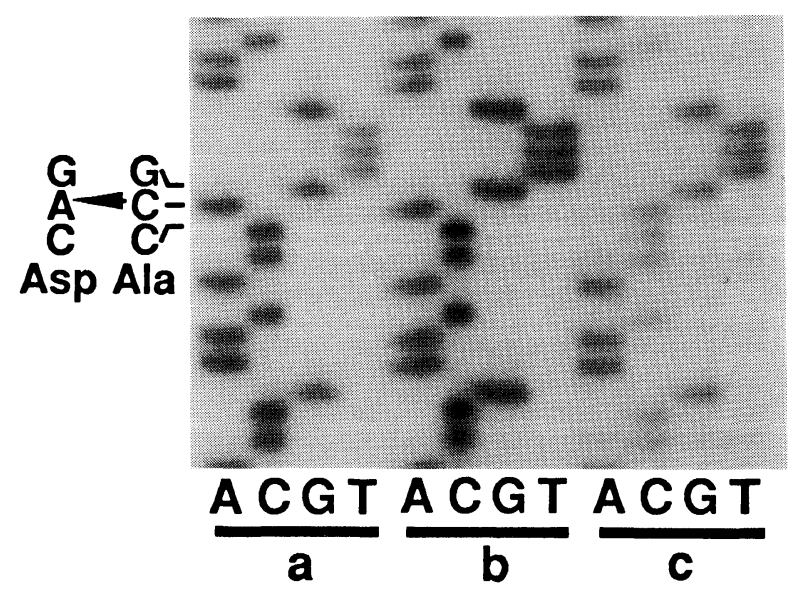

Fig. 2. A novel point mutation of the RET proto-oncogene in SCLC cell lines. Direct sequencing was performed with the sequencing primer as described in Materials and Methods. The arrows indicate the point mutation at codon 664 from GCC (Ala) to GAC (Asp) in Lu134A and Lu130 SCLC lines. Lanes from left to right are nucleotides of A, C, $\mathrm{G}$, and $\mathrm{T}$ of the sense strand. a, Lu134A; b, Lu130; c, PBMC from a normal volunteer.

Discussion. In the present study, we demonstrated that 2 out of 6 SCLC cell lines possessed a novel point mutation of the RET proto-oncogene, which substituted Asp (GAC) for Ala (GCC) at codon 664 in exon 11. The position of this point mutation is different from those associated with MEN2A, MEN2B, FMTC, sporadic MTC, sporadic pheochromocytoma, and Hirschsprung's disease (Fig. 3). In addition, the point mutation at codon 664 was not detected in 14 human cancer cell lines of other cancer types. These results suggest that this previously unknown point mutation is closely associated with a part of SCLC.

It is worth noting that PCR products of the normal $R E T$ proto-oncogene were not 


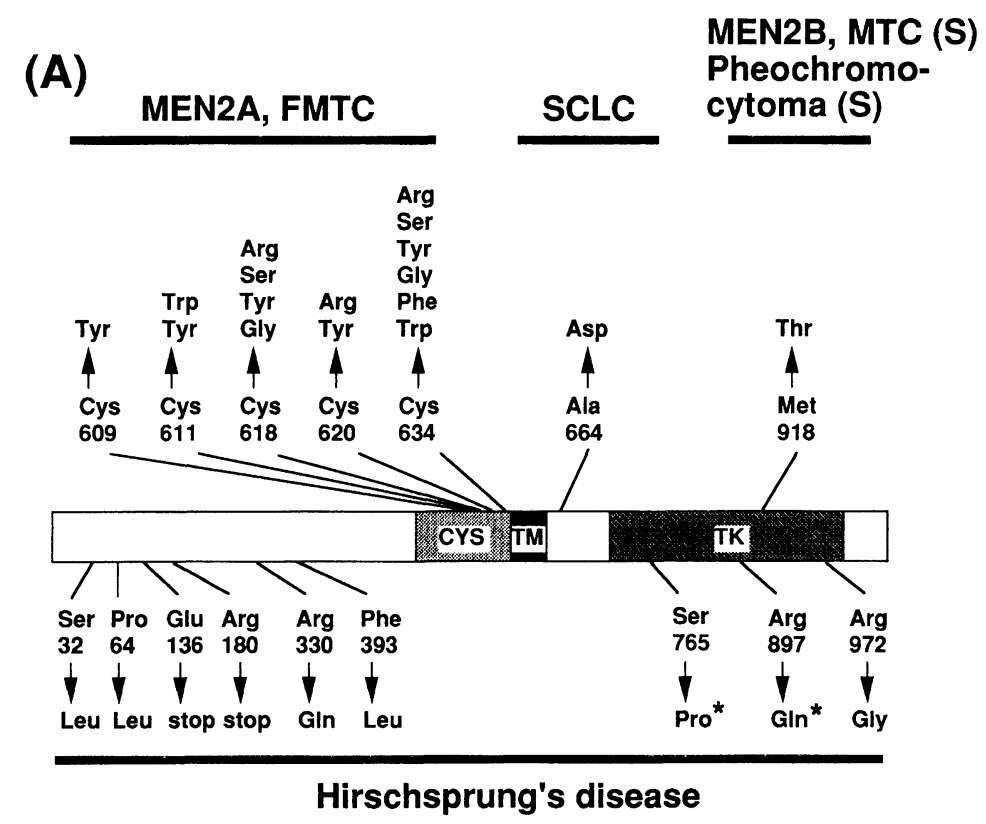

(B)

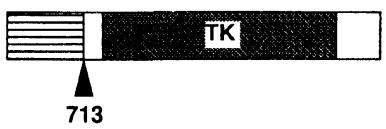

Fig. 3. Point mutations and a rearrangement of the RET proto-oncogene in various diseases. A, number of codons and amino acid substitutions in SCLC cell lines and various diseases are described. , sporadic Hirschsprung's disease; CYS, cysteine-rich region; S, sporadic; TM, transmembrane domain; TK, tyrosine kinase domain. $\mathrm{B}$, a rearrangement of the RET proto-oncogene in papillary thyroid carcinoma, ret ${ }^{\mathrm{TPC} /}$ $\mathrm{PTC},{ }^{4), 20)}$ is schematically described; a solid triangle and the number indicate the chimeric point of codon 713 at the $5^{\prime}$ end of exon 12 .

detected in either of these two cell lines that possessed mutant gene. It could be possible that this is due to allelic loss of the normal RET proto-oncogene, although a genetic polymorphism can not be completely excluded.

At any rate, it remains to be determined whether these genetic alterations had occurred during cell culture or had existed in the original tumor tissues from which these two cell lines were derived. In addition, whether they are of somatic or germ-line origin is unknown. Further studies are required to examine both of tumors and normal tissues obtained from SCLC patients for elucidating these points.

Acknowledgments. The authors would like to thank Dr. M. Nagao for her valuable comments, Drs. T. Tsukada and K. Sasaki for their helpful technical assistance, and Dr. T. Terasaki for providing SCLC and non-SCLC cell lines. This investigation was supported in part by a research grant from the Princess Takamatsu Cancer Research Fund, by a Grant-in-Aid from the Ministry of Health and Welfare for the 2nd-term Comprehensive 10-Year Strategy for Cancer Control, by Grants-in-Aid for Cancer Research (6-29) from the Ministry of Health and Welfare, and by the Special Coordination Funds from the 
Science and Technology Agency for Promoting Science and Technology.

\section{References}

1) Takahashi, M. et al. (1988): Oncogene, 3, 571-578.

2) Tahira, T. et al. (1990): ibid., 5, 97-102.

3) Ishizaka, Y. et al. (1989): Jpn. J. Cancer Res., 80, 1149-1152.

4) Grieco, M. et al. (1990): Cell, 60, 557-563.

5) Bongarzone, I. et al. (1993): Mol. Cell Biol., 13, 358-366.

6) Nagao, M. et al. (1990): Jpn. J. Cancer Res., 81, 309-312.

7) Ikeda, I. et al. (1990): Oncogene, 5, 1291-1296.

8) Donis-Keller, H. et al. (1993): Hum. Mol. Genet., 2, 851-856.

9) Mulligan, L. M. et al. (1993): Nature, 363, 458-460.

10) Hofstra, R. M. W. et al. (1994): ibid., 367, 375-376.

11) Eng, C. et al. (1994): Hum. Mol. Genet., 3, 237-241.

12) Edery, P. et al. (1994): ibid., 367, 378-380.

13) Romeo, G. et al. (1994): ibid., 367, 377-378.

14) Yamaguchi, K. et al. (1984): Metabolism, 33, 724-727.

15) Yamaguchi, K. et al. (1986): Jpn. J. Clin. Oncol., 16, 235-241.

16) Terasaki, T. et al. (1986): ibid., 16, 203-212.

17) Orita, M. et al. (1989): Genomics, 5, 874-879.

18) Ceccherini, I. et al. (1993): Biochem. Biophys. Res. Commun., 196, 1288-1295.

19) Itoh, F. et al. (1992): Oncogene, 7, 1201-1206.

20) Ishizaka, Y. et al. (1990): Biochem. Biophys. Res. Commun., 168, 402-408. 